\title{
A practical guide to effective behavior change: How to apply theory- and evidence-based behavior change methods in an intervention.
}

Serjo Kok

This document is the full text of the article "A practical guide to effective behavior change: How to apply theory- and evidence-based behavior change methods in an intervention" that has been published in the European Health Psychologist in 2014.

Although the European Health Psychologist is Open Access, it does not associate digital object identifiers to its publications. PsyArXiv does associate DOI's to the posted preprints, and in addition, PsyArXiv preprints are widely indexed. Therefore, this version has been published on PsyArXiv as well.

\section{Citing this manuscript}

To cite this manuscript, use the following citation:

Kok, G. (2014). A practical guide to effective behavior change: How to apply theoryand evidence-based behavior change methods in an intervention. European Health Psychologist, 16(5), 156-170. doi:10.31234/osf.io/r78wh

Other behaviour change resources

A number of behaviour change resources that may be useful to intervention developer or behaviour change researchers are available at https://effectivebehaviorchange.eu. In addition, the website of the Academy of Behavior Change, https://a-bc.eu, may be useful.

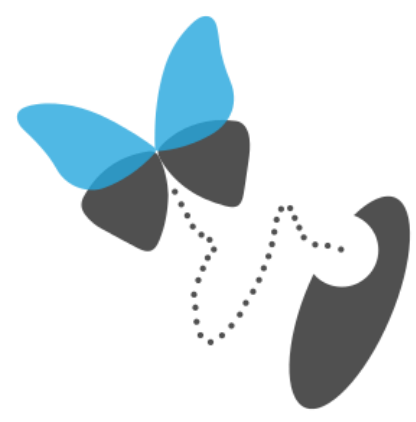




\section{original article}

\section{A practical guide to effective behavior change}

\section{How to apply theory- and evidence-based behavior change methods in an intervention}

\section{Gerjo Kok}

Maastricht University
In planning behavior

change, we encounter three major challenges: 1)

the correct identification of the change objectives (and thereby the evaluation outcomes), 2) the selection and application of appropriate behavior change methods in an intervention, and 3) adequate implementation of the intervention. As a consequence, the most frequent intervention failures include: incorrect identification of change objectives, inappropriate choice of methods or applications, or inadequate implementation in terms of completeness and fidelity of the program being delivered. The current contribution provides a practical guide to effective behavior change, with a particular focus on the second challenge: choosing behavior change methods, translating methods into practical strategies, and combining strategies in order to develop an effective intervention. This paper therefore fits well between the paper written by Peters (2014, this issue) on "How to identify what to change in the first place?", and the paper by Knittle (2014, this issue) on "Fidelity in intervention delivery".

\section{Intervention Mapping}

Our approach to intervention development is based on Intervention Mapping (IM). IM is a protocol for systematic theory-and evidence-based planning for behavior change (Bartholomew, Parcel, Kok, Gottlieb, \& Fernández, 2011). The IM protocol describes the iterative path from problem identification to problem solving or reduction. Each of the six steps of IM comprises several tasks, and each of these tasks

integrates theory and evidence. The completion of the tasks in each step creates an end product that can be used as a guide for the subsequent step. The completion of all of the steps serves as a blueprint for the design, implementation, and evaluation of an intervention that is based on a foundation of theoretical, empirical, and practical information. The six steps of the IM process are: (1) Conducting a needs assessment or problem analysis; (2) Creating matrices of change objectives by combining (sub)behaviors with behavioral determinants; (3) Selecting theory-based intervention methods and translating these into practical applications; (4) Integrating methods and applications into an organized program; (5) Planning for the adoption, implementation and sustainability of the program (from the start of the IM process); (6) Generating an evaluation plan (also from the start of the IM process). The key words in IM are planning, research and theory. IM provides a vocabulary for program planning, procedures for planning activities, and technical assistance with identifying theory-based determinants and matching them with appropriate methods for change. Of the three major planning challenges that were mentioned in the introduction, the first-- identifying the change objectives -comprises IM steps 1 and 2, and part of step 6. The second challenge--selecting the appropriate behavior change methods and applying those in an intervention--comprises IM steps 3 and 4, and the third challenge--adequate implementation--comprises IM step 5. Figure 1 summarizes the IM steps and tasks described above. 


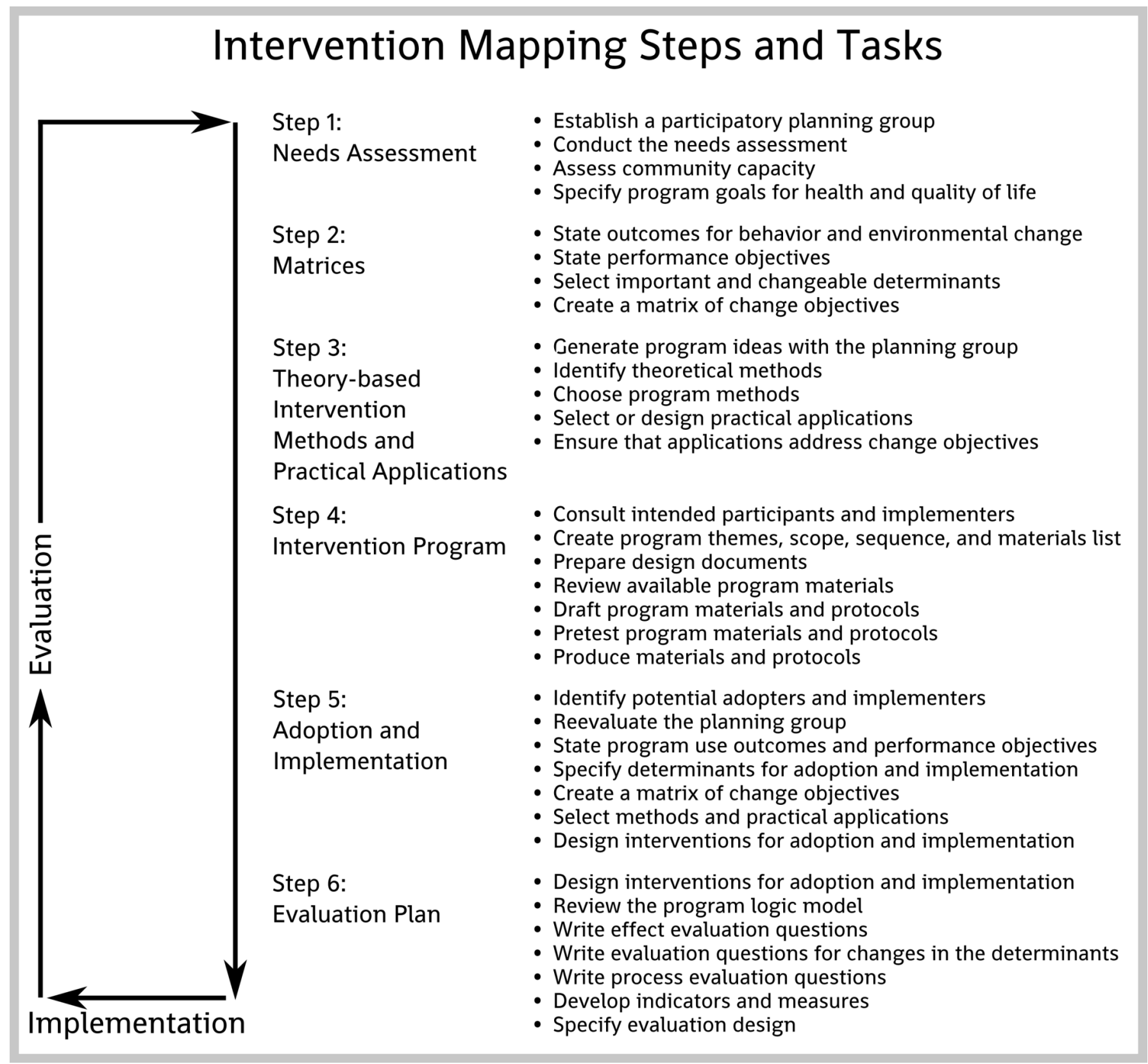

Figure 1: Intervention Mapping steps and tasks.

\section{Perspectives on theory, systems and participation}

IM is guided by three perspectives: a multi-theory approach, an ecological approach, and a participation perspective, each of which will be described in more detail below.

\section{The multi-theory approach}

IM encourages working with multiple theories. Theories can be seen as reductions of reality -- this is not a shortcoming, but rather the definition. One theory will therefore never explain all aspects of a real-life problem. As a consequence, we use various theories when planning behavior change, each of 
which focuses on one aspect of the behavior or the behavior change (Bartholomew et al., 2011, Chapters 2 and 3). Some theories are especially relevant in terms of identifying the determinants of behavior (e.g., Reasoned Action Approach, Social Cognitive Theory, Dual Systems Theory and Ecological Approach); others are more useful with regard to choosing and applying behavior change methods (see Table 1). One theory will seldom be enough to inform all aspects of the process. However, at the same time, it must be noted that attempting to integrate various theories into one overarching framework is rarely helpful. A theory is more than a list of variables; the relationship among the variables often forms the core of the theory. The unique skill of the well-trained behavioral scientist is to link the relevant elements of a given problem to useful theories (Buunk \& van Vugt, 2013). Ergo, behavioral scientists and their unique expertise are needed in an intervention planning team.

\section{The ecological approach}

As mentioned above, the second approach which informs IM processes is the ecological approach. IM acknowledges that humans and human behaviors are part of a complex system. Individuals live and work in many different kinds of multi-level environments, including interpersonal, organizational, community and societal environments (Bartholomew et al., 2011; Kok, Gottlieb, Commers, \& Smerecnik, 2008). Changing people's health behaviors (e.g. the behavior of a group of employees) therefore also involves changing the relevant environmental conditions (e.g. the workplace). These environments are often not under the control of the individual, but rather under the control of some agent or decision maker (e.g. a manager). Thus, changing an environmental condition for health purposes also involves changing the behavior of the environmental agent. On the one hand, environmental agents are individuals and may be targeted with individual-level behavior change methods. On the other hand, they also function at an environmental level, and may be targeted with behavior change methods that are effective at that level, e.g. organizational change methods or community development methods.

\section{The participation perspective}

The third approach that IM is guided by concerns the participation of all stakeholders (involved parties). IM sees planning health promotion interventions not as a solitary activity, but rather as team work. The participation of all stakeholders involved (including the target population) in the intervention planning team is not only a decent thing to do, but it is also essential for success. Earlier in this paper, we mentioned three possible major planning failures: incorrect identification of change objectives, inappropriate choice of methods or applications, and inadequate implementation. All three of these failures are often the result of insufficient participation of the target population (e.g. students), intended implementers (e.g. teachers), decision makers (e.g. school managers), and so forth. The only way to establish the relevant determinants of behavior, is by contacting the relevant target population, as well as the agents and stakeholders around them (see Box 1 and Box 2 in Peters, 2014, this issue). Similarly, the only way to find out how methods should be applied in the process is by contacting the target population, intended implementers and other relevant stakeholders.

\section{From change objectives to theory-based methods}

As mentioned earlier, Peters (2014, this issue) focuses on how to identify what needs to change (the first major challenge of the IM process), and ends his contribution with a matrix of change objectives for target individuals and environmental agents, the end product of IM step 2. As the next part of the process, 


\section{Box 1: Basic steps for linking change objectives to theory-based methods and practical applications}

Before you start:

A. Establish a participatory planning group and specify program goals. Ensure that there is a well-trained behavioral scientist in the planning team, as well as representatives of the target group and of the intended implementers.

B. Create a matrix of change objectives, see Peters (2014, this issue).

Basic steps:

1. Generate program ideas with the planning group. Most planning group members already have some ideas about the program. Planners must find a balance between preliminary ideas generated by team members on the one hand, and theory-and evidence-based decisions about methods, applications and programs on the other hand. What lay people think is effective may not be congruent with scientific evidence.

2. Identify theoretical methods. Order all change objectives by determinant, i.e. the columns in the matrix of change objectives, so that you end up with a list of change objectives (or, beliefs to influence) for each determinant. Then, determine which methods can change each determinant you have identified. Next, order the environmental change objectives according to their level (e.g. organizational, community, etc.). Determine which methods are appropriate for each level. Remember that individual level methods can also be applied to environmental level change objectives. Make sure you keep track of the parameters for effectiveness for each method, for example from the tables in Bartholomew, et al. (2011).

3. Choose program methods. On the basis of the lists of methods that you have compiled, select the methods you want to use. Ensure that all of the methods you select are theory-based. Again, keep in mind that all methods have parameters for effectiveness.

4. Select or design practical applications. Design creative program applications that fit the context and characteristics of the program participants while ensuring that the applications still address the parameters for the selected methods. When you are done, look through the lists of change objectives once again. Make sure that each change objective ended up in an application; that each application is the manifestation of one or several theoretical methods; and that all parameters of each method are satisfied.

we now need to link those change objectives to theoretical methods and apply those methods correctly in an intervention, IM steps 3 and 4 (see also Box 1).

A theory-based behavior change method is a general technique or process designed to influence the determinants of behavior (for example, of members of an at-risk group, or of environmental decision makers) (Abraham \& Michie, 2008;
Bartholomew et al., 2011). Theory-based methods are based on the literature regarding effective behavior change. This type of research almost never concerns methods for direct behavior change. Rather, in almost all cases, change methods are used to target determinants such as attitude or self-efficacy that are in turn thought to influence the behavior. In this way, theory-based methods are linked to change objectives via determinants. The generic nature of 
Table 1: A selection of methods, parameters, and examples of applications

\begin{tabular}{|c|c|c|}
\hline Methods, theory and definitions & Parameters for use & Examples \\
\hline \multicolumn{3}{|c|}{ Examples of basic methods at the individual level } \\
\hline $\begin{array}{l}\text { Modeling } \\
\text { Providing an appropriate model being } \\
\text { reinforced for the desired behavior. } \\
\text { (Social Cognitive Theory; Theories of } \\
\text { Learning: McAlister et al.,2008; Kazdin, } \\
\text { 2008) }\end{array}$ & $\begin{array}{l}\text { Attention, remembrance, self-efficacy } \\
\text { and skills, reinforcement of the model, } \\
\text { identification with the model, use of a } \\
\text { coping model instead of a mastery } \\
\text { model. }\end{array}$ & $\begin{array}{l}\text { The health promoter finds a role model } \\
\text { from the at-risk group who will } \\
\text { encourage identification and serve as a } \\
\text { coping model: } \\
\text { "I tried to quit smoking several times } \\
\text { and was not successful, then I tried ... } \\
\text { Now I have been off cigarettes for ..." }\end{array}$ \\
\hline
\end{tabular}

\section{Facilitation}

Creating an environment that makes the Requires real changes in the action easier or reduces barriers to action.

(Social Cognitive Theory: Bandura, 2004) environment, identification of barriers and facilitators, power for making changes, and usually intervention at a higher environmental level to facilitate conditions on a lower level.
A program that targets improvement in drug users' self-efficacy for using clean needles must also facilitate accessibility of clean needles.

\section{Examples of methods used to change awareness and risk perception}

\section{Consciousness raising}

Providing information, feedback, or confrontation about the causes, consequences of, and alternatives for, a problem or a problem behavior. (Health Belief Model; PrecautionAdoption Process Model; TransTheoretical Model: Champion \& Skinner, 2008; Weinstein et al., 2008;

Prochaska, et al., 2008)

\section{Scenario-based risk information}

Providing information that may aid the construction of an image of the ways in which a future loss or accident might occur.

(Precaution-Adoption Process

Model: Mevissen et al., 2009)
Can use feedback and confrontation. However, raising awareness must be quickly followed by an increase in problem-solving ability and (collective) self-efficacy.
An HIV counselor reminds a person of recent episodes of failure to use condoms when having sex, and the potential consequences of that behavior for significant others. Then the counselor encourages the planning of coping responses.
Plausible scenario with a cause and an Peer models in an HIV-prevention outcome; imagery. Most effective when program present a series of scenarios in people generate their own scenario or which they describe how they found when multiple scenarios are provided. themselves in risky situations, for example, a sexual relationship over the summer holidays. these determinants, and the methods used to change them, are consistent with the study of human behavior and psychology in general; yet at the same time, this means that such methods cannot immediately be applied in behavior change interventions. Rather, they require translation into practical applications (see next paragraph). With respect to environmental levels, methods are linked to each level: interpersonal, organizational, community and policy levels.

In order to select appropriate methods for changing environmental conditions in a health intervention, the first step is to find out who may be in a position to make the expected change. The program planner has to identify the desired behaviors for the agent who will actually change the environmental conditions in order to address the health issue at hand. The health promoter can then 
Table 1: A selection of methods, parameters, and examples of applications (continued)

\begin{tabular}{|c|c|c|}
\hline Methods, theory and definitions & Parameters for use & Examples \\
\hline \multicolumn{3}{|c|}{ Examples of methods used to change skills, capability, and self-efficacy, and to overcome barriers } \\
\hline $\begin{array}{l}\text { Cue altering } \\
\text { Changing a stimulus, either consciously } \\
\text { or unconsciously perceived, that elicits } \\
\text { or signals a behavior. }\end{array}$ & Existing positive intention. & $\begin{array}{l}\text { Dieters change the route they take, } \\
\text { walking to work in order to avoid easy } \\
\text { access to snack shops. }\end{array}$ \\
\hline
\end{tabular}

(Theories of Goal Directed Behavior;

Theories of Automatic, Impulsive and

Habitual Behavior: Verplanken \& Aarts,

1999; Wood \& Neal, 2007)

\section{Planning coping responses}

Prompting participants to list potential barriers and ways to overcome these. (Attribution Theory and Relapse Prevention Theory; Theories of Goal Directed Behavior: Marlatt \& Donovan, 2005; Hoffman et al., 2008)
Identification of high-risk situations and practice of coping responses.
The HIV nurse and the patient define the causes of non-adherence. Then the HIV nurse and the patient formulate solutions to solve or avoid the causes for non-adherence.

\section{Examples of basic methods at the environmental level}

\section{Participatory problem solving}

Diagnosing the problem, generating Requires willingness by the health potential solutions, developing priorities, promoter or convener to accept the making an action plan, and obtaining feedback after implementing the plan. (Organizational Development Theories; Social Capital Theory; Models of Community Organization: Butterfoss et al. 2008; Cummings \& Worley, 2015; Minkler et al. 2008)

\section{Technical assistance}

Providing the technical means to achieve Nature of technical assistance will vary desired behavior.

(Organizational Development Theories, Diffusion of Innovations Theory, Social Capital Theory, Models of Community Organization: Flaspohler et al., 2008; Mitchell et al., 2002) according to environmental level but must fit needs, culture, and resources of recipient.

\begin{abstract}
A health promotion consultant assists employees of a small company to identify the level and sources of stress and then develops a plan with management to address and monitor work stress.
\end{abstract}

\begin{abstract}
A health department liaison helps a community health center develop recruitment procedures, training, and supervisory guidelines as they establish a new lay health worker program.
\end{abstract}

apply methods to influence the determinants of the agent's behavior (using a variety of methods which are appropriate for changing determinants at different environmental levels). For example, a basic method used for changing determinants at all environmental levels is advocacy; a method used at the interpersonal level is enhancing network linkages, at the organizational level sense-making, at the community level social action, and at the policy level

\section{agenda setting.}

Most methods specifically target one type of determinant, e.g., scenario-based risk information is assumed to influence risk perception. It may also have a (weaker) effect on attitudes. Some methods are more generally applicable, e.g. modeling may be applied to influence self-efficacy, perceived norms, attitudes, or risk perception. Organizational diagnosis and feedback are most effective at the organizational 
Table 1: A selection of methods, parameters, and examples of applications (continued)

Methods, theory and definitions Parameters for use Examples

\section{Examples of methods used to change organizations}

Organizational diagnosis and

feedback

Assessing organizational structures and Methods appropriate to organizational An organizational consultant conducts employees' beliefs and attitudes, desired characteristics -- for example, size and a survey of employees' health behaviors outcomes and readiness to take action, information technology.

using surveys and other methods.

(Organizational Development Theory:

Cummings \& Worley, 2009) and determinants and holds focus groups consisting of employees to review the results and plan for health promotion programs.

\section{Increasing stakeholder influence}

Increase stakeholder power, legitimacy, The focal organization perceives that and urgency, often by forming coalitions the external organization or group is and using community development and one of its stakeholders.

social action to change an organization's policies.

(Stakeholder Theory) (Brown et al., 2003; Kok, et al., 2015; Mitchell et al.,

A community group uses media advocacy to highlight the groundwater pollution by gas storage tanks located in the community, and to demand that the tanks be moved by the gas company that owns them. 1997)

level. Participatory problem solving can be used at any level. In Table 1, we present some examples of behavior change methods, basic methods and methods per determinant or environmental level, definitions, theory-base, parameters (see next paragraph) and practical applications; adapted from Bartholomew et al. (2011; chapter 6 provides an overview of many theory-based methods).

\section{From theoretical methods to practical applications}

Practical applications are specific translations of theory-based methods for practical use. They should be tailored to the intervention population and the context in which the intervention will be conducted, and take the parameters for use into account (Bartholomew et al., 2011; see also Box 1). For example, change objectives for an intervention might focus on influencing adolescents' self-efficacy beliefs about always using condoms when having sex. The accompanying belief could, for example, be: "I am not very confident that I can always use condoms, when my partner does not want to". Successfully changing this belief would increase adolescents' self-efficacy (the generic determinant) to always use condoms: "I am confident that I can always use condoms, even when my partner does not want to". To achieve this change objective, theory-based methods might include modeling, guided practice with feedback, and reinforcement. One application of modeling in a school setting could be a videotaped step-by-step demonstration by similar adolescents of how to successfully convince an unwilling partner to use condoms, or how condom use can become more automatic. However, for a different population, such as intravenous drug-users, a booklet with carefully selected authentic modeling stories might be more appropriate (see Figure 2). Thus, the same method can be translated into a myriad of possible applications, depending on the specific population and context. Similarly, one application can be a manifestation of multiple methods (see Figure 3). Modeling applied in a school setting could improve self-efficacy and at the same time provide 


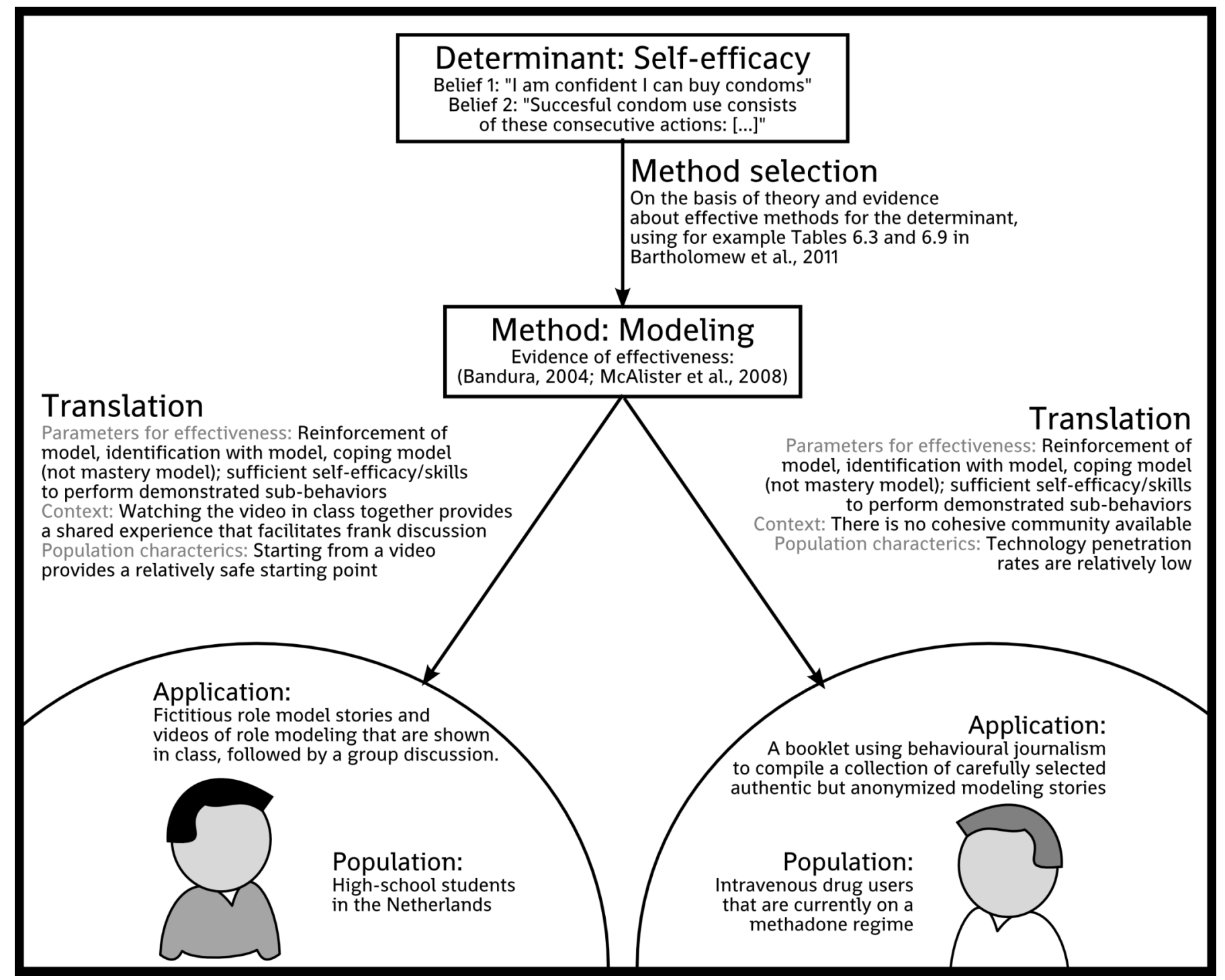

Figure 2: One method translated into different applications as a function of different populations and contexts.

information about the approval of others and change perceived norms.

The situation commonly encountered in the "real world" of intervention development (as compared to a tightly controlled research setting) is that theorybased methods tend to disappear in translation. In other words, even when there is a clear plan regarding which determinants to target, and which theory-based method to use, in the end, when translating these methods into actual materials and messages, some necessary methods are left out of the program Some methods may be lost in translation because logistical issues surrounding the development and production of program components and materials may become overwhelming, and so cuts are made to the plan. Other times, attempts are made to utilize theory-based change methods to influence each determinant, but the ways in which the practical applications are conceptualized and delivered do a poor job of translating the methods.

Translating methods into practical applications demands a sufficient understanding of the theory behind the method, especially the theoretical parameters which determine whether the theoretical 


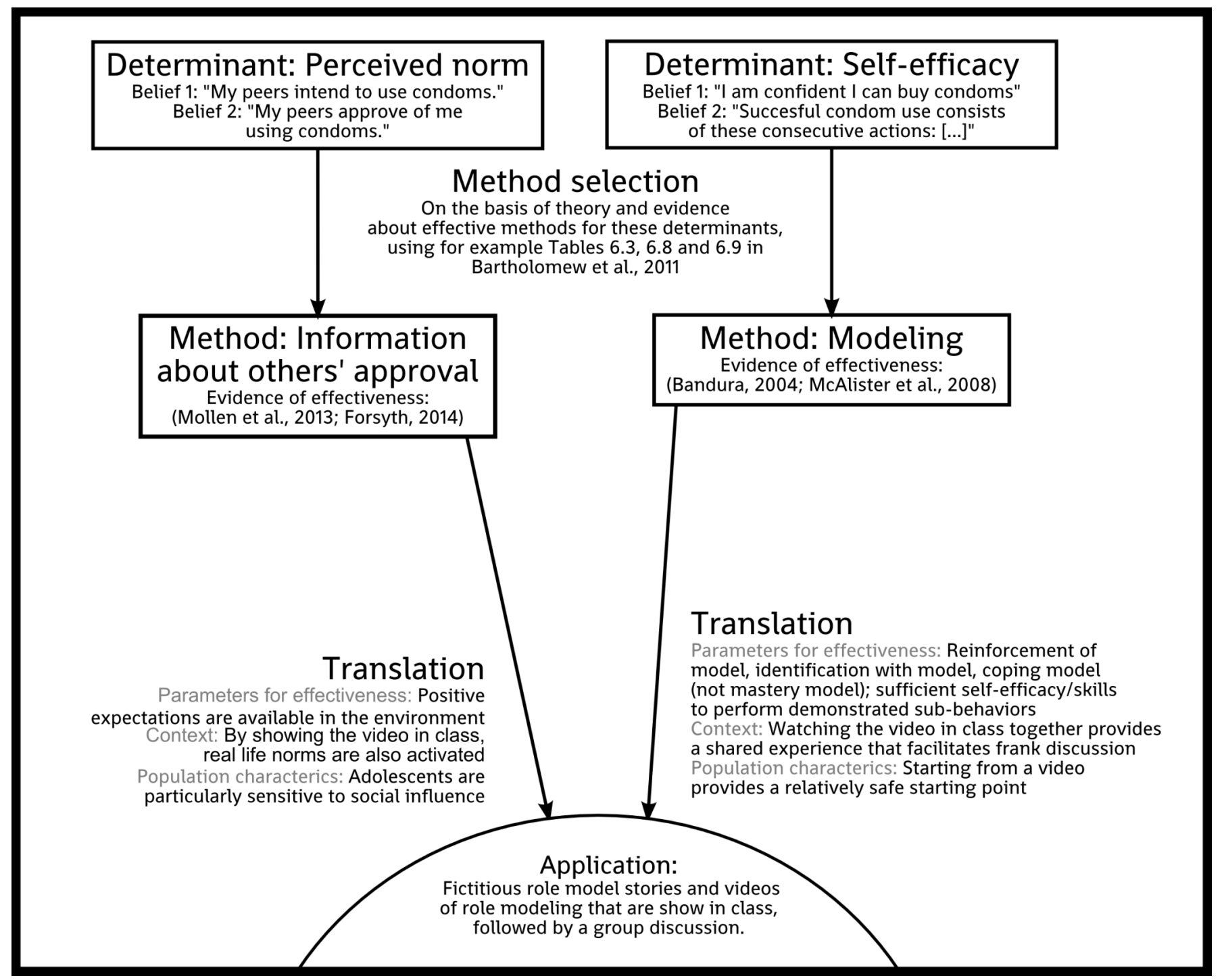

Figure 3: Two methods combined in one application.

process is effective or not (Kok, Gottlieb, Panne, \& Smerecnik, 2012; Schaalma \& Kok, 2009; Peters Ruiter, \& Kok, 2014). No method is always effective! For example, modeling is a strong and popular method but is only effective when certain parameters are met, for instance reinforcement of the modeled behavior (McAllister, Perry, \& Parcel, 2008). People or environmental decision makers do not imitate behavior simply because a model demonstrates that behavior; they behave in accordance with the model only when the model exhibits certain characteristics, such as being reinforced for that particular behavior (and they expect to be reinforced in a similar way). Translating the method modeling into a practical application necessitates taking care that in the actual program, from the perspective of the program participants, the model is reinforced. To provide a second example: goal setting can be a very effective method, but only when the goal is challenging as well as acceptable for the actor. People often choose goals outside those parameters. Moreover, fear appeals are only effective when the at-risk population has high (self-) efficacy, and they may actually be countereffective when efficacy is low (Peters, Ruiter, \& Kok, 
2013; Ruiter et al., 2014). Nevertheless, fear appeals are often inappropriately used (Peters et al., 2014; Ten Hoor et al., 2012). Behavior change is sometimes described as a two-step process involving 'motivation' and 'action' (Schwarzer, 2014). However, as a consequence of this, all theory-based methods that focus on 'action' presume that the target is already motivated, and yet this is not always the case. For example, implementation intentions are potentially very effective, but only when people have a positive intention in the first place; if not, implementation intentions will not be effective as a behavior change method. All theory-based methods have such parameters, which have to be taken into account when translating a method into a practical application. In Table 1, parameters are described for the examples of methods provided.

\section{From practical applications to an intervention}

So far, we have covered the various steps in the IM process. We have outlined perspectives on theory, systems, and participation, and described the journey from change objectives to theory-based methods, and how these methods are then translated into practical applications. How then, are these practical applications best integrated into an effective intervention?

"If you are not trained for something, don't do it" (Balderman, 1995). Essential in the collaboration with creative consultants is mutual respect: respect the creative professional, but also ensure that the creative professional respects the behavioral scientist's competence. Creative consultants are seldom aware of the parameters for effectiveness that apply to methods, and it is the responsibility of the behavioral scientist to make sure that those parameters will stay intact. Always return to the matrices of change objectives and the lists of methods, parameters and applications (see Box 2).
A program theme is a general overarching construct for a program, sometimes organized into sub-themes. Examples of themes include: the Active Plus exercise program for the over-fifties (van Stralen et al., 2008), the Gay Cruise safe sex program for internet dating MSM (Kok et al., 2006), or Cultivando la Salud, a lay health worker intervention to increase breast and cervical cancer screening among lowincome Hispanic women (Fernandez et al., 2009). A theme should be attractive to the target population and might also already affect relevant determinants, e.g.: Watch, Discover, Think and Act (Bartholomew et al., 2000). The scope refers to the breadth and size of the program, describing what is and what is not in the program: for example, how much do we focus on topics such as abortion or sexual diversity in a school-based sex education program? The sequence refers to the order in which the elements of a program are delivered across time. Communication channels can be interpersonal or mediated; vehicles refer more specifically to how messages are packaged and delivered; each option has advantages and disadvantages. Peer education can be a powerful source of persuasion, is often inexpensive, and involves the community. However, peer educators can be difficult to train and to keep motivated. Entertainment-education via television has the benefit of a wide distribution and has norm changing capabilities. However, the influence of TV-producers with different objectives can be extremely difficult to counter (Bartholomew et al., 2011, Chapter 7).

Sometimes, existing materials may be useful. Still, new or existing materials need to be matched with the previously developed matrices of change objectives and the lists of methods, parameters and applications (especially the parameters for use, the appropriateness for the target group, and the context). The planning group needs to ensure that communication will go back-and-forth with the production group regarding all materials and products. All materials and products need to be pilot tested. Creative consultants, as well as managers or funders, have a tendency to suggest immediate 


\section{Box 2: Basic steps for integrating applications into an intervention}

Before you start:

A. Establish a participatory planning group and specify program goals. Ensure that there is a well-trained behavioral scientist in the planning team, as well as representatives of the target group and of the intended implementers.

B. Create a matrix of change objectives, see Peters (2014, this issue).

C. Select or design practical applications, see Box 1.

Basic steps:

1. Consult intended participants and implementers. Avoid simplistic thinking by staying focused on the end products of the previous planning steps: program goals, change objectives, applications. Allow creativity to flourish. Respect the input from the target group, and respect any cultural differences. When ideas for the intervention setting take form, invite intended implementers into the planning team.

2. Create program themes, scope, sequence, and materials list. Specify program scope and sequence, describe each population group and program interface, and include a list of program materials and staff required for that interface. Describe the program budget for materials production.

3. Prepare design documents. Hire creative consultants and make sure that mutual respect is guaranteed. Talk about and agree what the creative people will return in various forms. Keep the matrix of change objectives available as well as the lists of methods, parameters and applications.

4. Review available program materials. Match existing materials against matrices of change objectives and lists of methods, parameters and applications. Determine suitability, availability and appropriateness of reading level.

5. Draft program materials and protocols. Ensure a back-and-forth interaction between the planning team and the production team. Observe budget limits and respect cultural differences.

6. Pretest and revise program materials. Resist all proposals to skip pretesting, check for parameters, and use experimental designs, if possible.

7. Produce materials and protocols. Oversee the final production.

implementation, with the argument that the program has been developed carefully. However, there are enough examples of well-developed programs that turn out to be unintentionally counterproductive to insist on pretesting. If possible, apply experimental designs in pretests (Whittingham et al., 2009).

\section{From the intervention to the implementation}

Once the intervention has been created, a solid diffusion and implementation process is vital to ensure program success. Without implementation, the intervention will not have any impact on determinants, behaviors, or health. So, in IM Step 5, a plan is developed for the systematic implementation of the program. The first thing to do, actually right at the start of intervention development, is to develop a 
linkage system, linking program developers with program users in the planning team. Next, an intervention is developed to promote adoption and implementation of the program by the intended program users. Intervention planners develop strategies to facilitate the implementation of the health promotion intervention with high fidelity and completeness. They develop theory-based strategies to facilitate program adoption by key stakeholders, to support appropriate implementation by program users, and to encourage program institutionalization by considering opportunities for incorporating the program into organizational routines. Thus, interventions are not only required to change individual behavior, but also to facilitate program implementation. Indeed, the same steps involved in intervention development are repeated to anticipate program diffusion and to target program implementers. Sustainable implementation almost always involves organizational change, for example in a school setting (Hendriks et al., 2013). See also the contribution of Knittle (2014, this issue).

\section{Conclusion}

Behavior change is extremely difficult to plan. If behavior change was easy, it would have already happened; professional health promotion planners become engaged when all simpler interventions to change behavior have failed and the desired behavior changes are extremely difficult to accomplish. An optimal approach has a higher chance of success, but success is never guaranteed. The highest chance for success can be expected from a theory- and evidencebased process. In this paper, we described IM as one such protocol. Essential to the IM process is the correct identification of the change objectives, followed by the selection of the appropriate behavior change methods, and the application of those in an intervention. Lastly, care must be taken that the intervention is adequately implemented. In this paper, we described the second challenge in detail: applying theory- and evidence-based behavior change methods in an intervention promoting healthy behaviors and environments. Much of what we wrote is about logically and professionally applying the methods of behavioral science. To sum up with the most important message: methods are only effective when applied within their theory- and evidence-based parameters. In order to do this, well-trained behavioral scientists need to be involved in the planning process: everything should be as simple as possible, but no simpler (Peters, et al., 2013).

\section{References}

Abraham, C., \& Michie, S. (2008). A taxonomy of behavior change techniques used in interventions. Health Psychology, 27(3), 379-387. doi:10.1037/0278-6133.27.3.379

Balderman, B. (1995). Buying Creative Services. Lincolnwood, IL: NTC Publishing Group.

Bandura, A. (2004). Health promotion by social cognitive means. Health Education and Behavior, 31(2), 143-164. doi:10.1177/1090198104263660

Bartholomew, L. K., Gold, R. S., Parcel, G. S., Czyzewski, D. I., Sockrider, M. M., Fernandez, M., ... Swank, P. (2000). "Watch, Discover, Think, and Act": Evaluation of computer-assisted instruction to improve asthma self-management in inner-city children. Patient Education and Counseling, 39(2), 269-280. doi:10.1016/S0738-3991(99)00046-4

Bartholomew, L. K., Parcel, G. S., Kok, G., Gottlieb, N. H., \& Fernández, M.E. (2011). Planning health promotion programs; an Intervention Mapping approach (3rd ed.) San Francisco, CA: Jossey-Bass.

Brown, L. D., Bammer, G., Batliwala, S., \& Kunreuther, F. (2003). Framing practice-research engagement for democratizing knowledge. Action Research, 1(1), 81-102. doi:10.1177/14767503030011006

Butterfoss, F. D., Kegler, M. C., \& Francisco, V. T. (2008). Mobilizing organizations for health promotion: Theories of organizational change. In K. Glanz, B. K. Rimer, \& K. Viswanath (Eds.), Health behavior and health education: Theory, 
research, and practice (4th ed.) (pp. 335-362). San Francisco: Jossey-Bass.

Buunk, A.P., \& van Vugt, M. (2013). Applying Social Psychology: From Problems to Solutions. London: Sage.

Champion, V. L., \& Skinner, C. S. (2008). The Health Belief Model. In K. Glanz, B. K. Rimer, \& K. Viswanath (Eds.), Health behavior and health education: Theory, research, and practice (4th ed., pp. 45-65). San Francisco, CA: Jossey-Bass.

Cummings, T. G., \& Worley, C. G. (2015). Organization development and change (10th ed.). Mason, $\mathrm{OH}$ : South-Western Cengage Learning.

Fernandez, M. E., Gonzales, A., Tortolero-Luna, G., Williams, J., Saavedra-Embesi, M., Chan, W., \& Vernon, S. W. (2009). Effectiveness of Cultivando la Salud: A breast and cervical cancer screening promotion program for low-income Hispanic women. American Journal of Public Health, 99(5), 936-943. doi:10.2105/AJPH.2008.136713

Flaspohler, P., Duffy, J., Wandersman, A., Stillman, L., \& Maras, M. A. (2008). Unpacking prevention capacity: An intersection of research-to-practice models and community-centered models. American Journal of Community Psychology, 41(3-4), 182-196. doi:10.1007/s10464-008-9162-3

Forsyth, D.H. (2014). Group dynamics (6th ed.). Wadsworth: Cengage Learning.

Hendriks, A. M., Jansen, M. W. J., Gubbels, J. S., de Vries, N. K., Paulussen, T., \& Kremers, S. P. J. (2013). Proposing a conceptual framework for integrated local public health policy, applied to childhood obesity - the behavior change ball. Implementation Science, 8(1), 46-63. doi:10.1186/1748-5908-8-46

Hofmann, W., Friese, M., \& Wiers, R. W. (2008). Impulsive versus reflective influences on health behavior: A theoretical framework and empirical review. Health Psychology Review, 2(2), 111-137. doi:10.1080/17437190802617668

Kazdin, A. E. (2008). Behavior modification in applied settings (6 ed.). Long Grove, IL: Waveland Press.

Knittle, K. (2014). Fidelity in intervention delivery: A rough field guide. The European Health
Psychologist, 16(5), 190-195.

Kok, G., Harterink, P., Vriens, P., De Zwart, 0., \& Hospers, H. J., (2006). The Gay Cruise: Developing theory- and evidence-based Internet HIVprevention. Sexuality Research and Social Policy, 3(2), 52-67. doi:10.1525/srsp.2006.3.2.52

Kok, G., Gottlieb, N. H., Commers. M., \& Smerecnik, C. (2008). The ecological approach in health promotion programs; A decade later. American Journal of Health Promotion, 22(6), 437-442. doi:10.4278/ajhp.22.6.437

Kok, G., Gottlieb, N. H., Panne, R., \& Smerecnik, C. (2012). Methods for environmental change; an exploratory study. BMC Public Health, 12, 1037. doi:10.1186/1471-2458-12-1037

Kok, G., Gurabardhi, Z., Gottlieb, N.H., \& Zijlstra, F. (2015). Influencing organizations to promote health: Applying stakeholder theory. Manuscript submitted for publication.

Marlatt, G. A., \& Donovan, D. M. (Eds.) (2005). Relapse prevention; maintenance strategies in the treatment of addictive behaviors (2nd ed.). New York: Guilford.

McAlister, A. L., Perry, C. L., \& Parcel, G. S. (2008). How individuals, environments, and health behaviors interact: social cognitive theory. In K. Glanz, B. K. Rimer, \& K. Viswanath (Eds), Health Behavior and Health Education (4th ed) (pp. 169-88). San Francisco, CA: Jossey-Bass.

Mevissen, F. E. F., Meertens, R. M., Ruiter, R. A. C., Feenstra, H., \& Schaalma, H. P. (2009). HIV/STI risk communication: The effects of scenario-based risk information and frequency-based risk information on perceived susceptibility to chlamydia and HIV. Journal of Health Psychology, 14(1), 78-87. doi:10.1177/1359105308097948

Mitchell, R. K., Agle, B. R., \& Wood,D. J. (1997).Toward a theory of stakeholder identification and salience: Defining the principle of who and what really counts. Academy of Management Review, 22(4), 853-886. doi:10.5465/AMR.1997.9711022105

Mitchell, R. E., Florin, P., \& Stevenson, J. F. (2002). Supporting community-based prevention and 
health promotion initiatives: Developing effective technical assistance systems. Health Education and Behavior, 29(5), 620-639. doi:10.1177/109019802237029

Mollen, S., Rimal, R. N., Ruiter, R. A. C., Jang, S. A., \& Kok, G. (2013). Intervening or interfering? The influence of injunctive and descriptive norms on intervention behaviours in alcohol consumption contexts. Psychology \& Health, 28(5), 561-578. doi:10.1080/08870446.2012.752827

Peters, G.-J. Y. (2014) . A practical guide to effective behavior change: How to apply theory- and evidence-based behavior change methods in an intervention. The European Health Psychologist, 16(5), 142-155.

Peters, G.-J. Y., de Bruin, M., \& Crutzen, R. (2013). Everything should be as simple as possible, but no simpler: towards a protocol for accumulating evidence regarding the active content of health behaviour change interventions. Health Psychology Review. Advance online publication. doi:10.1080/17437199.2013.848409

Peters, G. -J. Y., Ruiter, R. A. C., \& Kok, G. (2013). Threatening communication: A critical re-analysis and a revised meta-analytic test of fear appeal theory. Health Psychology Reviews, 7(sup1), S8S31. doi: 10.1080/17437199.2012.703527

Peters, G.-J. Y., Ruiter, R. A. C., \& Kok, G. (2014). Threatening communication: A qualitative study of fear appeal effectiveness beliefs among intervention developers, policy makers, politicians, scientists, and advertising professionals. International Journal of Psychology, 49(2), 71-79. doi:10.1002/ijop.12000

Prochaska, J. 0., Redding, C. A., \& Evers, K. E. (2008). The Transtheoretical Model and stages of change. In K. Glanz, B. K. Rimer, \& K. Viswanath (Eds.), Health behavior and health education: Theory, research, and practice (4th ed.) (pp. 97-121). San Francisco: Jossey-Bass.

Ruiter, R. A., Kessels, L. T., Peters, G. J. Y., \& Kok, G. (2014). Sixty years of fear appeal research: Current state of the evidence. International Journal of Psychology, 49(2), 63-70. doi:10.1002/ijop.12042
Schaalma, H. \& Kok, G. (2009). Decoding health education interventions: The times are a-changin'. Psychology \& Health, 24(1), 5-9. doi:10.1080/08870440903126348

Schwarzer, R. (2014). Health behavior change. Theoretical constructs, dynamic mechanisms and clinical interventions. In: S. Cooper \& K. Ratele (Eds.), Psychology serving humanity (pp. 140-154). New York: Psychology Press.

Shegog, R., Bartholomew, L. K., Sockrider, M. M., Czyzewski, D. I., Pilney, S., Mullen, P. D., \& Abramson, S. L. (2006). Computer-based decision support for pediatric asthma management: description and feasibility of the Stop Asthma Clinical System. Health Informatics Journal, 12(4), 259-273. doi:10.1177/1460458206069761

ten Hoor, G. A., Peters, G.-J. Y., Kalagi, J., de Groot, L., Grootjans, K., Huschens, A., ... Kok, G. (2012). Reactions to threatening health messages. BMC Public Health, 12, 1011. doi:10.1186/1471-245812-1011

van Stralen, M. M., Kok, G., de Vries, H., Mudde, A. N., Bolman, C., \& Lechner, L. (2008). The Active plus protocol: Systematic development of two theory- and evidence-based tailored physical activity interventions for the over-fifties. BMC Public Health, 8, 399. doi:10.1186/1471-2458-8399.

Verplanken, B., \& Aarts, H. (1999). Habit, attitude, and planned behaviour: is habit an empty construct or an interesting case of goal-directed automaticity? European Review of Social Psychology, 10(1), 101-134. doi:10.1080/14792779943000035

Weinstein, N. D., Sandman, P. M., \& Blalock, S. J. (2008). The Precaution Adoption Process Model. In K. Glanz, B. K. Rimer, \& K. Viswanath (Eds.), Health behavior and health education: Theory, research, and practice (4th ed.) (pp. 123-165). San Francisco: Jossey-Bass.

Whittingham, J., Ruiter, R., Bolier, L., Lemmers, L., van Hasselt, N., \& Kok, G., (2009). Avoiding counterproductive results: An experimental pretest of a harm reduction intervention on attitude 
toward party drugs among users and nonusers.

Substance Use \& Misuse, 44(4), 532-547.

doi:10.1080/10826080802347685

Wood, W., \& Neal, D. T. (2007). A new look at habits and the habit-goal interface. Psychological Review, 114(4), 843-863. doi:10.1037/0033-

295X.114.4.843

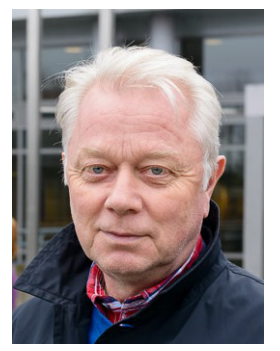

\section{Gerjo Kok}

Maastricht University, The

Netherlands

g.kok@ maastrichtuniversity.nl 